\title{
Acute administration of diosgenin or dioscorea improves hyperglycemia with increases muscular steroidogenesis in STZ-induced type 1 diabetic rats
}

\author{
Koji Sato ${ }^{1}$ Ph.D, Satoshi Fujita ${ }^{1}$ Ph.D, Motoyuki Iemitsu ${ }^{1} \mathrm{Ph} . D$ \\ ${ }^{1}$ Faculty of Sport and Health Science, Ritsumeikan University, Kusatsu, Shiga, \\ Japan.
}

Running title: Diosgenin improves hyperglycemia in type 1 diabetic rats

Address for reprint requests and correspondence: Motoyuki Iemitsu, Ph.D.

TEL: +81-77-599-4131

FAX: +81-77-561-3761

Mailing address: 1-1-1 Nojihigashi, Kusatsu, Shiga, Japan. 525-8577

E-mail: iemitsu@fc.ritsumei.ac.jp 


\section{Abstract}

Acute dehydroepiandrosterone (DHEA) administration improves hyperglycemia in rats with streptozotocin (STZ)-induced type 1 diabetes mellitus. Diosgenin, a steroid structurally similar to DHEA (dehydroepiandrosterone), is contained highly levels in dioscorea; however, it is still unclear whether this natural product improves hyperglycemia in the type 1 diabetes model rats through an increase muscular GLUT4 signaling. After 1 week of STZ injection, fasting glucose level was measured in blood taken from the tail vein every $30 \mathrm{~min}$ for $150 \mathrm{~min}$ after injection of diosgenin or dioscorea $(3 \mathrm{mg} / \mathrm{kg})$. On another day, muscle was resected $150 \mathrm{~min}$ after diosgenin or dioscorea injections. Serum DHEA level increased significantly 120 min after diosgenin or dioscorea injections; concomitantly, blood glucose level decreased significantly. Moreover, GLUT4 translocation, as well as phosphorylation of Akt and $\mathrm{PKC} \zeta / \lambda$, increased significantly by diosgenin or dioscorea administration. However, these effects of diosgenin and dioscorea were blocked by a $5 \alpha$-reductase inhibitor that inhibits synthesizing dehydrotestosterone (DHT) from testosterone. Additionally, significant correlations were observed between blood glucose level, GLUT4 translocation level, and muscular sex steroid hormone level 150 min after the administrations. These results suggest that the diosgenin-induced increase in the DHEA level may contribute to the 
improvement of hyperglycemia by activating the muscular GLUT4 signaling pathway in type 1 diabetes model rats.

Key words: natural nutrients, glucose transporter-4, protein kinase B, protein kinase $\mathrm{C}$ $\zeta / \lambda$ 


\section{Introduction}

Dioscorea, a plant widely known as wild yam, contains high levels of the natural product diosgenin. Diosgenin, a steroid sapogenin, is the product of hydrolysis of saponins by acids, strong bases, or enzymes, and can be extracted from the tubers of Dioscorea esculenta. Diosgenin has a steroid molecular formula similar to that of dehydroepiandrosterone (DHEA) (1). DHEA, a precursor of sex steroid hormones, is converted to testosterone and estradiol. Previously, we demonstrated that increased levels of sex steroid hormone resulting from DHEA administration contribute to improve hyperglycemia in type 1 and type 2 diabetic model rats $(2,3)$. In several previous studies, administration of dioscorea exerted various effects including alleviation of osteoporosis (4) and hyperlipidemia (5) and improvement of neuroimmunological function (6). However, it is still unclear whether the administration of dioscorea, which contains high levels of diosgenin, replenishes DHEA concentration in vivo and affects glycemic control under hyperglycemic conditions.

Recently, we demonstrated that skeletal muscle can locally synthesize testosterone and 5 $\alpha$-dihydrotestosterone (DHT) from DHEA; DHT exerts more biophysiological activities than testosterone $(7,8)$. In another study, the addition of DHEA to cultured skeletal muscle induced upregulation of GLUT4 protein via increased activation of 
protein kinase B (Akt) and protein kinase $\mathrm{C}-\zeta / \lambda(\mathrm{PKC} \zeta / \lambda)(8)$. Additionally, a single bout of DHEA administration for streptozotocin (STZ)-induced type 1 diabetic model rats improved hyperglycemia through the activation of the Akt and PKC $\zeta / \lambda$-GLUT-4 signaling pathway, and also increased DHEA and DHT in the skeletal muscle (2). Therefore, the sex steroid hormones, such as DHEA, regulate glucose utilization in skeletal muscles, and may participate in glycemic control of hyperglycemia. However, it is still unknown whether diosgenin and dioscorea administration to type 1 diabetic model rats improves hyperglycemia through the DHEA-induced activation of muscular Akt and PKC $\zeta / \lambda$-GLUT-4 signaling.

We hypothesized that a single bout of diosgenin or Dioscorea esculenta (lesser yam) injection results in conversion of diosgenin to DHEA, and that this replenishment activates the GLUT4-regulated signaling pathway in skeletal muscle, consequently improving hyperglycemia of STZ-induced type 1 diabetic rats. To test this hypothesis, we first investigated whether administration of diosgenin, which has a chemical structure similar to that of DHEA, would increase sex steroid hormone levels in serum and muscular tissue levels. Second we investigated whether dioscorea, which contains high levels of diosgenin, would increase these sex steroid hormone levels. Moreover, we monitored blood glucose levels before and after each injection of diosgenin or 
dioscorea, and examined Akt and PKC $\zeta / \lambda$ phosphorylation and GLUT4 translocation in the skeletal muscle. Finally, we investigated whether the acute effect of a single bout of diosgenin or dioscorea injection on hyperglycemia and activation of the muscular GLUT4-regulated signaling pathway would be attenuated by an inhibitor that blocks the conversion of testosterone to DHT. 


\section{Methods}

\section{Animals and protocol}

Ethical approval for this study was obtained from the Committee on Animal Care at the Ritsumeikan University. Male Wistar rats (aged 14 weeks) were obtained (Charles River Japan Inc., Kanagawa, Japan) and cared for according to The Guiding Principles for the Care and Use of Animals, based on the Declaration of Helsinki. These rats were maintained on a 12:12-h light-dark cycle and received food and water ad libitum. At 14 weeks of age, the animals were assigned randomly to three groups: diabetes control with sesame oil $(n=10)$, diabetes with diosgenin injection (Wako Pure Chemical Industries Ltd., Osaka, Japan) $(n=10)$, and diabetes with diosgenin injection and $5 \alpha$-reductase inhibitor $(n=10)$. To detect the acute effect of the plant source of diosgenin, dioscorea esculenta (lesser yam) was administered to two other groups: diabetes with dioscorea injection $(n=10)$ and diabetes with dioscorea injection and $5 \alpha$-reductase inhibitor $(n=10)$. Based on previous studies, $10 \mathrm{mg}$ of diosgenin (Sigma Aldrich Co, LLC, St Louis, MO) and $1 \mathrm{~g}$ of dioscorea powder (Takara Health Care, Kyoto, Japan) dissolved in sesame oil was injected intraperitoneally into the animals $(1,9,10,11)$. In the $5 \alpha$-reductase inhibitor group, $1 \mathrm{mg}$ of $5 \alpha$-reductase inhibitor (dutasteride; Sigma, Steinheim, Germany), dissolved in sesame oil, was injected intraperitoneally $1 \mathrm{~h}$ before 
diosgenin or dioscorea injection.

Type 1 diabetes mellitus was induced by a single intraperitoneal injection of STZ (Sigma Chemical Co., St. Louis, MO, USA). STZ was dissolved in 0.1 M sodium citrate buffer $(\mathrm{pH} \mathrm{4.5)}$, and injected at a dosage of $55 \mathrm{mg} / \mathrm{kg}$. Weight was checked before and after STZ injection. One week after STZ injection, the fasting glucose concentration was determined in a blood sample taken from the tail vein. Diabetes was defined as fasting blood glucose levels greater than $19.4 \mathrm{mmol} / \mathrm{L}$, as in previous studies $(12,13)$. The blood glucose level was monitored (Bayer Health Care, Tokyo, Japan) every $30 \mathrm{~min}$ for $180 \mathrm{~min}$ after diosgenin, dioscorea, or vehicle injection. After another 2 days, diosgenin, dioscorea, or vehicle was again injected, and the gastrocnemius muscle was resected quickly, rinsed in ice-cold saline, weighed, and frozen in liquid nitrogen. The muscle resection took place 90 min after diosgenin or dioscorea injection, when blood glucose levels are maximally reduced by these agents.

\section{Immunoblot analysis}

The muscle specimen was homogenized with $20 \mathrm{mM}$ Tris- $\mathrm{HCl}$ (pH 7.8), $300 \mathrm{mM}$ $\mathrm{NaCl}, 2 \mathrm{mM}$ ethylenediaminetetraacetic acid (EDTA), $2 \mathrm{mM}$ dithiothreitol (DTT), 2\% Nonidet P-40 (NP-40), 0.2\% sodium lauryl sulfate (SDS), $0.2 \%$ sodium deoxycholate, 
$0.5 \mathrm{mM}$ phenylmethylsulfonyl fluoride (PMSF), $60 \mu \mathrm{g} / \mathrm{ml}$ aprotinin, and $1 \mu \mathrm{g} / \mathrm{ml}$ leupeptin. The homogenate was rotated gently for $30 \mathrm{~min}$ at $4{ }^{\circ} \mathrm{C}$, and then centrifuged at $12,000 \times g$ for $15 \mathrm{~min}$ at $4^{\circ} \mathrm{C}$. The protein concentration of the resulting supernatant was determined. Samples $\left(50 \mu \mathrm{g}\right.$ protein) were denatured at $96^{\circ} \mathrm{C}$ for $7 \mathrm{~min}$ in Laemmli buffer. Western-blot analysis was performed to detect phosphorylated Akt and $\mathrm{PKC} \zeta / \lambda$, as well as GLUT4 protein expression (3). Briefly, each sample was separated on a $10 \%$ SDS-polyacrylamide gel and transferred to a polyvinylidene difluoride (PVDF; Millipore Corp., Billerica, MA, USA) membrane. The membrane was treated with blocking buffer, 5\% skim milk in phosphate-buffered saline with $0.1 \%$ Tween 20 (PBS-T), for $24 \mathrm{~h}$ at $4^{\circ} \mathrm{C}$. The membrane was probed with polyclonal anti-GLUT4 (Millipore Corp., Billerica, MA, USA), anti-serine (Ser) 473-phosphorylated Akt, anti-Akt, or anti-phosphorylated PKC $\zeta / \lambda$ antibody (Cell Signaling, Beverly, MA); all antibodies were diluted 1:1000 in blocking buffer (8). The membrane was washed three times with PBS-T, and then incubated for $1 \mathrm{~h}$ at room temperature with a horseradish peroxidase (HRP)-conjugated secondary antibody, anti-rabbit immunoglobulin, diluted 1:3000 in blocking buffer (GE Healthcare Biosciences, Piscataway, NJ, USA and Cell Signaling, Beverly, MA, USA). The membrane was then washed three times with PBS-T. Finally, GLUT4, phosphorylated Akt, total Akt, and phosphorylated PKC $\zeta / \lambda$ 
proteins were detected using an enhanced chemiluminescence system (ECL Plus; GE Healthcare Biosciences, Piscataway, NJ, USA) and visualized using an Image Quant LAS 4000 (GE Healthcare Biosciences).

\section{Preparation of the cytosolic and plasma membrane protein fractions}

To assess GLUT-4 translocation, two membrane fractions were prepared as previously described (3). Briefly, to prepare the crude membrane, muscles were scraped into buffer A (20 mM Tris [pH 7.4], 1 mM EDTA, 0.25 mM EGTA, $0.25 \mathrm{M}$ sucrose, 1 $\mathrm{mM}$ DTT, $50 \mathrm{mM}$ NaF, $25 \mathrm{mM}$ sodium pyrophosphate, and $40 \mathrm{mM}$ $\beta$-glycerophosphate). The resulting homogenates were clarified by centrifugation at 400 $\times g$ for $15 \mathrm{~min}$. The supernatant was centrifuged at $100,000 \times g$ for $1 \mathrm{~h}$. The enriched GLUT-4 membrane fraction was produced by blending muscles five times for $20 \mathrm{~s}$ in ice-cold Tris buffer containing 1.4 M sucrose and 0.25 mM PMSF. The homogenate was centrifuged for $10 \mathrm{~min}$ at $1,500 \times g$ at $4^{\circ} \mathrm{C}$. The postnuclear supernatant was added to 1 $\mathrm{mM}$ EDTA and prepared for density centrifugation by overlaying the postnuclear supernatant with Tris buffer containing 1.2 and $0.8 \mathrm{M}$ sucrose. The enriched GLUT-4 membrane fraction was harvested in the 0.8/1.2 $\mathrm{M}$ sucrose interphase. Two hundred microliters of the harvested interphase sample was resuspended in buffer A. To prepare 
the cytosolic protein fraction, cells were solubilized for $1 \mathrm{~h}$ at room temperature in buffer B (20 mM Tris [pH 7.4], 1 mM EDTA, $70 \mathrm{mM} \mathrm{KCl,} 3 \mathrm{mM}$ magnesium acetate, 2 $\mathrm{mM} \mathrm{CaCl} 2$ ). The homogenate was centrifuged, and the supernatant was spun for $1 \mathrm{~h}$ at $100,000 \times g$. The resulting supernatant was then used as the cytosolic fraction. GLUT-4 protein levels were measured in both the membrane and cytosolic fractions. Translocation was determined as the difference in protein levels between the cytosol and membrane fractions (3).

\section{Enzyme-linked immunosorbent assay (ELISA)}

The levels of DHEA and DHT in skeletal muscle extracts and serum were determined using an enzyme immunoassay for the diagnostic quantitative determination of DHEA (Enzo Life Sciences Inc., Farmingdale, NY) and DHT (IBL International, Hamburg, Germany). All techniques and materials used in these analyses were in accordance with the manufacturer's protocol. Cross-reactivities, as listed in the assay description, were $100 \%$ for DHEA and it was less than $0.5 \%$ cross reactivity for any other related steroid compounds such as $0.10 \%$ for testosterone, $0.058 \%$ for progesterone and $0.037 \%$ for estradiol. All samples were assayed in duplicate. Optical density at $450 \mathrm{~nm}$ was qualified using a microplate reader (xMark microplate 
spectrophotometer; Bio-Rad, Tokyo, Japan). In addition, the serum insulin concentration was measured using an ELISA Kit (Shibayagi Co. Ltd., Gunma, Japan) according to the manufacturer's protocol. All samples were assayed in duplicate. Optical density at 420 nm was qualified using a microplate reader (xMark microplate spectrophotometer; Bio-Rad).

\section{Statistical analysis}

All values are expressed as means \pm SE. Statistical evaluations for blood glucose levels were performed using ANOVA for repeated measures (time $\times$ group). A post-hoc comparison test was used to correct for multiple comparisons (Bonferroni test) when analyses revealed significant differences. For this ANOVA, $P<0.01$ was considered significant. Other data were analyzed using one-way ANOVA. Again, a post-hoc comparison test was used to correct for multiple comparisons when analyses revealed significant differences. For one-way ANOVA, $P<0.05$ was considered significant. Relationships between sex steroid hormone concentrations and serum insulin, blood glucose levels, and activation of glucose metabolism signaling were determined using Pearson correlation coefficients. 


\section{Results}

Animal characteristics

No differences in body weight were observed among the groups (STZ control, diosgenin or dioscorea injection, and diosgenin or dioscorea injection with 5a-reductase inhibitor). Serum insulin levels were significantly lower in STZ rats than in normal rats. The fasting blood glucose level was significantly higher in STZ rats (Table 1).

Blood glucose level

Blood glucose level was significantly decreased 90-180 min after diosgenin injection, relative to the control group and diosgenin and $5 \alpha$-reductase inhibitor group.

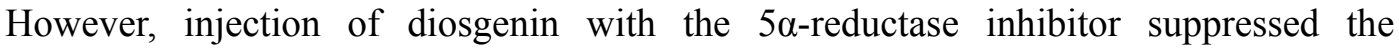
diosgenin-induced decrease in blood glucose level (Fig. 1A). Likewise, dioscorea injection also significantly decreased blood glucose level at 90-180 min after injection, compared to the control group, and at 150-180 min after injection compared to the $5 \alpha$-reductase inhibitor group, whereas injection of dioscorea with the $5 \alpha$-reductase inhibitor blocked the dioscorea-induced decrease in blood glucose level (Fig. 2A). 
Serum DHEA concentration was significantly increased 120 min after diosgenin injection, and 150 min after dioscorea injection (Fig. 2A). Serum DHT concentration was also significantly increased $150 \mathrm{~min}$ after diosgenin or dioscorea injection (Fig. 2B).

Muscular DHEA concentrations were significantly greater in rats subjected to diosgenin injection and diosgenin injection with $5 \alpha$-reductase inhibitor than in the control rats (Fig. 3A). Muscular DHT concentrations were significantly greater in the diosgenin injection rats than in control rats. However, the DHT concentrations were significantly lower in the $5 \alpha$-reductase inhibitor rats than in the diosgenin injection rats (Fig. 3A). The muscular concentrations of DHEA and DHT in the muscle were confirmed, which corrected by per g tissue. The data was not different from the corrected value by $\mu \mathrm{g}$ protein.

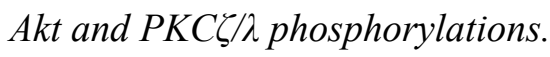

Akt phosphorylation was greater in diosgenin injection rats than in STZ control rats. However, the effect of diosgenin on Akt phosphorylation was attenuated in the $5 \alpha$-reductase inhibitor injected rats. Similarly, dioscorea injection induced a significant increase in Akt phosphorylation, whereas 5a-reductase inhibitor suppressed this 
dioscorea-induced increase, so no significant difference was observed between STZ control rats and $5 \alpha$-reductase inhibitor rats (Fig. 4A, B). In fact, PKC $\zeta / \lambda$ phosphorylation in skeletal muscle was significantly lower in STZ control rats, but significantly higher in diosgenin injection rats (Fig. 4A). On the other hand, the effect of diosgenin was attenuated in the $5 \alpha$-reductase inhibitor rats. Dioscorea injection induced a significant increase in $\mathrm{PKC} \zeta / \lambda$ phosphorylation, whereas $5 \alpha$-reductase inhibitor suppressed the dioscorea-induced increase of $\mathrm{PKC} \zeta / \lambda$ phosphorylation, so there was no significant difference in $\mathrm{PKC} \zeta / \lambda$ phosphorylation between $5 \alpha$-reductase inhibitor rats and STZ control rats (Fig. 4A, B).

\section{GLUT4 protein expression and its translocation}

GLUT4 protein expression in skeletal muscle was significantly lower in STZ control rats (Fig. 5A), but higher in the diosgenin injection rats than in the STZ control rats. The effect of DHEA was attenuated in 5 $\alpha$-reductase inhibitor rats (Fig. 5A). Moreover, the GLUT4 protein levels in both cytosol and the plasma membrane of skeletal muscle were higher in the diosgenin injection rats than in the STZ control rats (Fig. 5A). GLUT4 translocation, determined from the difference in cytosol and membrane GLUT-4 protein levels, was significantly lower in STZ control rats than in 
the normal control rats (Fig. 5A), but significantly higher in the diosgenin injection rats than in STZ control rats. However, the effect of diosgenin on GLUT4 translocation was significantly attenuated in the 5 $\alpha$-reductase inhibitor rats (Fig. 5A). Dioscorea injection also significantly increased GLUT4 protein levels, as well as GLUT4 translocation, in both the cytosol and the plasma membrane in skeletal muscle (Fig 5B). On the other hand, the effect of dioscorea on GLUT4 translocation was significantly suppressed in the $5 \alpha$-reductase inhibitor rats (Fig. 5B).

Relationship between sex steroid hormone concentrations, GLUT4 translocation level, and blood glucose level Significant negative correlations were observed between GLUT4 translocation level and blood glucose level at $150 \mathrm{~min}$ after diosgenin or dioscorea administration $(\mathrm{r}=$ -0.825, $P<0.01)$. Moreover, muscular sex steroid hormones were significantly correlated with both GLUT4 translocation level and blood glucose levels (GLUT4 translocation: $\mathrm{r}=0.541, P<0.05$, blood glucose level: $\mathrm{r}=-0.612, P<0.05$; Table 2). 


\section{Discussion}

In this study, a single bout of diosgenin or dioscorea increased serum DHEA and concomitantly improved hyperglycemia in STZ-induced type 1 diabetes model rats. Moreover, these effects of diosgenin or dioscorea were blocked by using an inhibitor of the enzyme involved in DHT synthesis. Diosgenin has a chemical structure similar to that of DHEA, and it may increase serum sex steroid hormone levels; this compound is contained highly levels in dioscorea (1). In type 1 diabetic rats, which have lower levels of plasma sex steroid hormones, treatment of DHEA by diosgenin or dioscorea administration may contribute to the improvement of hyperglycemia. Thus, natural products such as diosgenin (or dioscorea, a rich source of this compound) may be helpful in glycemic control in the type 1 diabetes.

In this study, diosgenin or dioscorea administration induced a significant increase in muscular sex steroid hormone levels, and significantly activated the muscular Akt and PKC $\zeta / \lambda$-GLUT4 signaling pathways in type 1 diabetes mellitus model rats. Moreover, this study confirmed that diosgenin or dioscorea effects could be attenuated by a $5 \alpha$-reductase inhibitor. Recently, we demonstrated DHEA-induced improvement of hyperglycemia through the activation of muscular Akt and PKC $\zeta / \lambda$-GLUT4 signaling pathways in type 1 diabetes model rats (2). In this study, diosgenin- or 
dioscorea-induced improvement of plasma glucose levels was associated with muscular DHT levels and GLUT4 translocation levels. Thus, diosgenin and dioscorea increase muscular levels of sex steroid hormone, i.e., DHT, and this hormone may contribute to improvement of hyperglycemia through upregulation of Akt and PKC $\zeta / \lambda$-GLUT4 signaling pathways in skeletal muscle tissues of rats with type 1 diabetes.

The results of this study showed that diosgenin and dioscorea administration induced a significant increase in serum DHEA level after $120 \mathrm{~min}$; DHEA and DHT levels peaked after $150 \mathrm{~min}$. In rats with type 1 diabetes mellitus, diosgenin and dioscorea administration decreased blood glucose level by about $28 \%$ and $21 \%$, respectively. In our previous study, a 29\% decrease in blood glucose level was seen 90 min after DHEA injection, compared to the baseline level (2). The effect of diosgenin or dioscorea administration may be delayed relative to the effects of DHEA administration, because diosgenin and dioscorea must be converted to DHEA in vivo. In this study, administration of either diosgenin or dioscorea in conjunction with a $5 \alpha$-reductase inhibitor blocked the decrease of blood glucose level. In addition, diosgenin or dioscorea administration increased muscular Akt and $\mathrm{PKC} \zeta / \lambda$ phosphorylation by $30 \%$, and increased GLUT4 translocation by about $30 \%$, relative to untreated type 1 diabetic rats. These activations are similar to DHEA administration (2). Thus, diosgenin and 
dioscorea induced a decrease in blood glucose level, and activated the muscular GLUT4 signaling pathway, to the same degree as DHEA administration. We speculate that administration of diosgenin or dioscorea should also replenish sex steroid hormones and improve hyperglycemia in vivo.

Although diosgenin and dioscorea administration increased serum sex steroid hormone levels in STZ-induced diabetes rats, it is still unclear where in the body the diosgenin and dioscorea are converted to DHEA. This issue should be addressed in a future study. Patients with type 1 diabetes mellitus depend on insulin injections or insulin-pump treatment, and they require careful self-testing of blood glucose levels in order to prolong their lives and prevent diabetes-related complications. Poorly controlled type 1 diabetes mellitus is a risk factor for coronary artery disease, renal failure, and retinopathy (14). It is crucially important to treat these patients and prevent these complications. Future studies should focus on the effect of chronic diosgenin or dioscorea administration on glycemic control in patients with type 1 diabetes. Regarding the mechanism of these effects, diosgenin and dioscorea administration induced increases in both serum and muscle sex steroid hormone, and circulating sex steroid hormone improved hyperglycemia by activating glucose metabolism signaling pathway. If intake of natural products were to affect glucose uptake and utilization in 
skeletal muscle, these hormones would be helpful for controlling blood glucose levels in type 1 diabetes mellitus. In this study, the dose of diosgenin was adapted from the dose used for humans in a previous study. As noted above, diosgenin is present at high levels in dioscorea. By LC/MS analysis, $1 \mathrm{~g}$ dioscorea contains about $9.5 \mathrm{mg}$ of diosgenin (data not shown). In the previous study, $3 \mathrm{~g}$ of diosgenin was administered to humans (1); thus, $120 \mathrm{~g}$ of dioscorea might be the effective dose for humans. Therefore, the doses of diosgenin and dioscorea used in this study were within physiological range in the present study.

In summary, this study demonstrated that a single bout of injection of diosgenin, which is present at high levels in dioscorea, elevated serum and muscular DHEA levels and concomitantly decreased blood glucose levels in type 1 diabetes model rats. Furthermore, diosgenin alleviated the STZ-induced decline in the Akt and PKC $\zeta / \lambda$-GLUT4 pathways, and a 5 $\alpha$-reductase inhibitor blocked these effects. Consequently, the natural product diosgenin is a candidate for use in acute improvement of blood glucose level and restoration of impaired muscular glucose metabolism regulation in skeletal muscle in type 1 diabetes mellitus. 


\section{Acknowledgements}

This work was supported by Grants-in-Aid for Scientific Research from the Ministry of Education, Culture, Sports, Science and Technology of Japan (23700849, 25560378, 23680071). This work was also funded by The Yamaha Motor Foundation for Sports (K. Sato).

\section{Disclosure}

The authors have no conflicts of interest to declare. 


\section{References}

1. Raju J, Patlolla JM, Swamy MV, Rao CV. Diosgenin, a steroid saponin of Trigonella foenum graecum (Fenugreek), inhibits azoxymethane-induced aberrant crypt foci formation in F344 rats and induces apoptosis in HT-29 human colon cancer cells, 2004, 13:1392-1398.\#

2. Sato K, Iemitsu M, Aizawa K, Ajisaka R. DHEA improves impaired activation of Akt and PKC $\zeta / \lambda$-GLUT4 pathway in skeletal muscle and improves hyperglycaemia in streptozotocin-induced diabetes rats. Acta Physiol, 2009, 197: 217-225.

3. Sato K, Iemitsu M, Aizawa K, Mesaki N, Fujita S. Increased muscular dehydroepiandrosterone levels are associated with improved hyperglycemia in obese rats. Am J Physiol, 2011, 301: E274-E280.

4. Chiang SS, Chang SP, Pan TM. Osteoprotective effect of monascus-fermented dioscorea in ovariectomized rat model of postmenopausal osteoporosis. J Agric Food Chem, 2011, 59:9150-9157.

5. Komesaroff PA, Black CV, Cable V, Sudhir K. Effects of wild yam extract on menopausal symptoms, lipids and sex hormones in healthy menopausal women. Climacteric, 2001, 4:144-150.

6. Ying $\mathrm{JH}$, Ching $\mathrm{FW}$, Wen $\mathrm{YH}$, Ting $\mathrm{T}$, Cheng $\mathrm{CH}$, Mei DK, Yuan FT. 
Psychoimmunological effects of dioscorea in ovariectomized rats. Ann Gen Psychiatry. 2007, 10;6:21.

7. Aizawa K, Iemitsu M, Maeda S, Jesmin S, Otsuki T, Mowa CN, Miyauchi T, Mesaki N. Expression of steroidogenic enzymes and synthesis of sex steroid hormones from DHEA in skeletal muscle. Am J Physiol. 2007, 292: E577-E584.

8. Sato K, Iemitsu M, Aizawa K, Ajisaka R. Testosterone and DHEA activate the glucose metabolism-related signaling pathway in skeletal muscle. Am J Physiol. 2008, 294:E961-E968.

9. Coleman DL. Schwizer RW. Leiter EH. Effect of genetic background on the therapeutic effects of dehydroepiandrosterone (DHEA) in diabetes-obesity mutants and in aged normal mice. Diabetes. 1984, 33:26-32.

10. Enoki T., Yoshida Y., Hatta H., Bonen A. Exercise training alleviates MCT1 and MCT4 reductions in heart and skeletal muscles of STZ-induced diabetic rats. J Appl Physiol. 2003, 94:2433-2438.

11. Ishizuka T., Miura A., Kajita K., Matsumoto M., Sugiyama C., Matsubara K., Ikeda T., Mori I., Morita H., Uno Y., Mune T., Kanoh Y., Ishizawa M. Effect of dehydroepiandrosterone on insulin sensitivity in Otsuka Long-Evans Tokushima-fatty rats. Acta Diabetol. 2007, 44:219-226.

12. Dimitrakoudis D., Vranic M., Klip A. Effects of hyperglycemia on glucose transporters of the muscle: Use of the renal glucose reabsorption inhibitor phlorizin 
to control glycemia. J Am Soc Nephrol. 1992, 3:1078-1091.

13. Wilson D., Chase HP., Kollman C., Xing D., Caswell K., Tansey M., Fox L., Weinzimer S., Beck R., Ruedy K., Tamborlane W., The Diabetes Research in Children Network (DirecNet) Study Group. Low-fat vs. high-fat bedtime snacks in children and adolescents with type 1 diabetes. Pediatr Diabetes. 2008, 28:320-325.

14. Hagiwara H., Kaizu K., Uriu K., Noguchi T., Takagi I., Qie YL., Seki T., Ariga T. Expression of type-1 plasminogen activator inhibitor in the kidney of diabetic rat models. Thromb Res. 2003, 111:301-309. 


\section{Figure Legends}

Figure 1: Effect of diosgenin (A) or dioscorea (B) administration on plasma glucose levels in type 1 diabetes mellitus model rats.

Black triangle: STZ-control $(n=10)$. Black circle: STZ rats treated with diosgenin or dioscorea $(n=10)$. Black square: STZ rats treated with diosgenin or dioscorea and a $5 \alpha$-reductase inhibitor $(n=10)$.

Data are means $\pm \mathrm{SE}$.

$* P<0.01$ compared with the STZ-control; $\dagger P<0.05$ compared with $5 \alpha$-reductase inhibitor injection; DIO: diosgenin administration. Dios: dioscorea administration.

Figure 2: Effect of diosgenin (A) or dioscorea (B) administration on serum sex steroid hormone levels in type 1 diabetes mellitus model rats.

Data are means $\pm \mathrm{SE}$.

* $P<0.01$ compared with the STZ-control.

DIO: diosgenin administration. Dios: dioscorea administration.

Figure 3: Effect of diosgenin (A) or dioscorea (B) administration on muscular sex steroid hormone levels in type 1 diabetes mellitus model rats.

Data are means $\pm \mathrm{SE}$.

* $P<0.01$ compared with the STZ-control. DIO: diosgenin administration. Dios: dioscorea administration.

Figure 4: Effect of diosgenin (A) or dioscorea (B) on Akt and protein kinase C (PKC) $\zeta / \lambda$ phosphorylation in the gastrocnemius muscle. 
Representative images of immunoblotting for phosphorylated Akt and total Akt protein are shown in the upper panels. The lower panel shows the results of statistical analyses of the ratio of phosphorylated Akt protein to total Akt protein expression. The ratio of Akt phosphorylation in skeletal muscle was calculated by dividing the level of phosphorylated Akt protein by the total Akt protein level. p-Akt, phosphorylated Akt. Representative images of immunoblotting for phosphorylated protein-kinase C (PKC) $\zeta / \lambda$ protein are shown in the upper panel. The lower panel shows the results of statistical analyses of the level of phosphorylated protein-kinase $\mathrm{C}(\mathrm{PKC}) \zeta / \lambda$ expression. Phospho-PKC, phosphorylated protein kinase C.

Data are means $\pm \mathrm{SE}$.

$* P<0.01$ compared with the STZ-control; $\dagger P<0.05$ compared with $5 \alpha$-reductase inhibitor injection.

DIO: diosgenin administration. Dios: dioscorea administration.

Figure 5: Effect of diosgenin (A) or dioscorea (B) administration on glucose transporter-4 (GLUT4) protein expression and its translocation in the gastrocnemius muscle.

Representative images of immunoblotting for GLUT4 protein expression are shown in the upper panel. The lower panel shows the results of statistical analyses of the level of GLUT4 protein expression, obtained using densitometry, and representative images of immunoblotting for GLUT4 proteins in cytosol and the membrane are depicted in the upper panels. The lower left panel presents the results of statistical analyses of the level of GLUT4 protein expression, determined by densitometry. The each right panel shows the level of GLUT4 translocation, calculated from the difference in GLUT4 protein 
levels between cytosol and membrane fractions.

Data are means $\pm \mathrm{SE}$.

* $P<0.01$ compared with the control; $\uparrow P<0.05$ compared with $5 \alpha$-reductase inhibitor injection. 
Table 1: Animal characteristics

\begin{tabular}{|c|c|c|c|c|c|}
\hline & $\begin{array}{c}\text { Control } \\
(\mathrm{n}=10)\end{array}$ & $\begin{array}{c}\text { DIO } \\
(\mathrm{n}=10)\end{array}$ & $\begin{array}{c}\text { DIO + in } \\
(\mathrm{n}=10)\end{array}$ & $\begin{array}{c}\text { Dios } \\
(\mathrm{n}=10)\end{array}$ & $\begin{array}{c}\text { Dios + in } \\
(\mathrm{n}=10)\end{array}$ \\
\hline $\begin{array}{c}\text { Body mass } \\
(\mathrm{g})\end{array}$ & $302.4 \pm 4.1$ & $313.3 \pm 5.5$ & $324.5 \pm 8.1$ & $321.4 \pm 5.2$ & $318.6 \pm 7.2$ \\
\hline $\begin{array}{c}\text { Muscle weight } \\
\text { (g/body mass) } \\
\text { Gastrocnemius }\end{array}$ & $1.9 \pm 0.2$ & $2.0 \pm 0.3$ & $2.2 \pm 0.4$ & $1.8 \pm 0.4$ & $2.1 \pm 0.3$ \\
\hline $\begin{array}{c}\text { Fasting glucose } \\
(\mathrm{mg} / \mathrm{dl})\end{array}$ & $420.1 \pm 9.2$ & $412.4 \pm 8.5$ & $429.1 \pm 6.2$ & $416.5 \pm 7.1$ & $425.7 \pm 9.2$ \\
\hline $\begin{array}{c}\text { Serum insulin } \\
(\mathrm{pmol} / \mathrm{l})\end{array}$ & $0.34 \pm 0.2$ & $0.41 \pm 0.3$ & $0.47 \pm 0.4$ & $0.44 \pm 0.4$ & $0.36 \pm 0.5$ \\
\hline
\end{tabular}

DIO: diosgenin administration; DIO + in: diosgenin administration and $5 \alpha$-reductase inhibitor treatment

Dios: dioscorea administration; Dios +in: dioscorea administration and $5 \alpha$-reductase inhibitor treatment 
Table 2: Association of systemic and local hormone levels with GLUT4 translocation and blood glucose level

\begin{tabular}{|l|c|c|c|c|}
\hline & \multicolumn{2}{|c|}{ GLUT4 translocation } & \multicolumn{2}{c|}{ Blood glucose } \\
\hline $\begin{array}{l}\text { Muscle } \\
\text { hormones }\end{array}$ & $\mathrm{r}$ & $\mathrm{P}$ & $\mathrm{r}$ & $\mathrm{P}$ \\
\hline DHEA & -0.521 & & & $<0.05$ \\
\hline DHT & -0.541 & $<0.05$ & 0.544 & $<0.05$ \\
\hline
\end{tabular}


Figure 1

Sato et al.

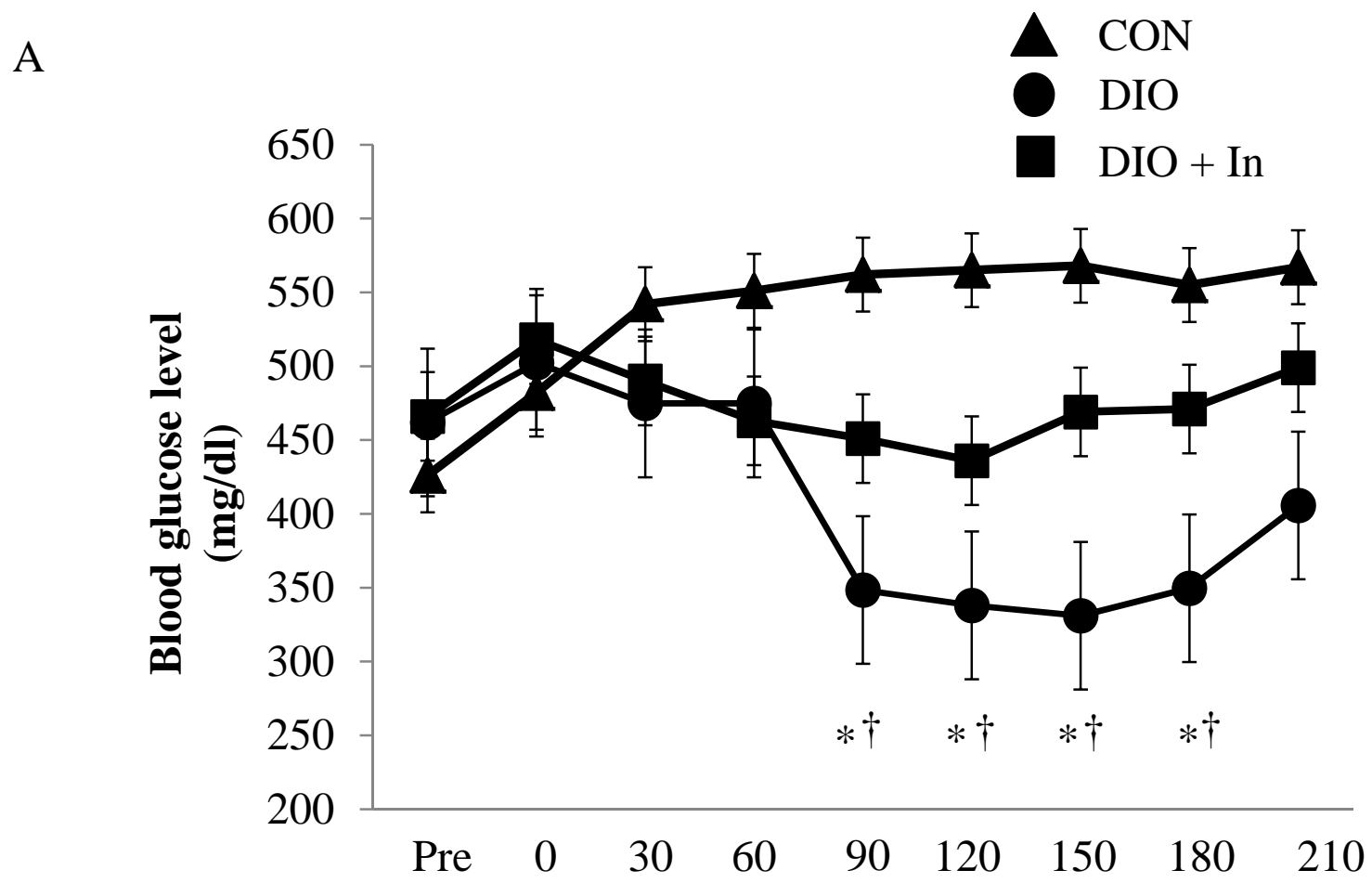

B

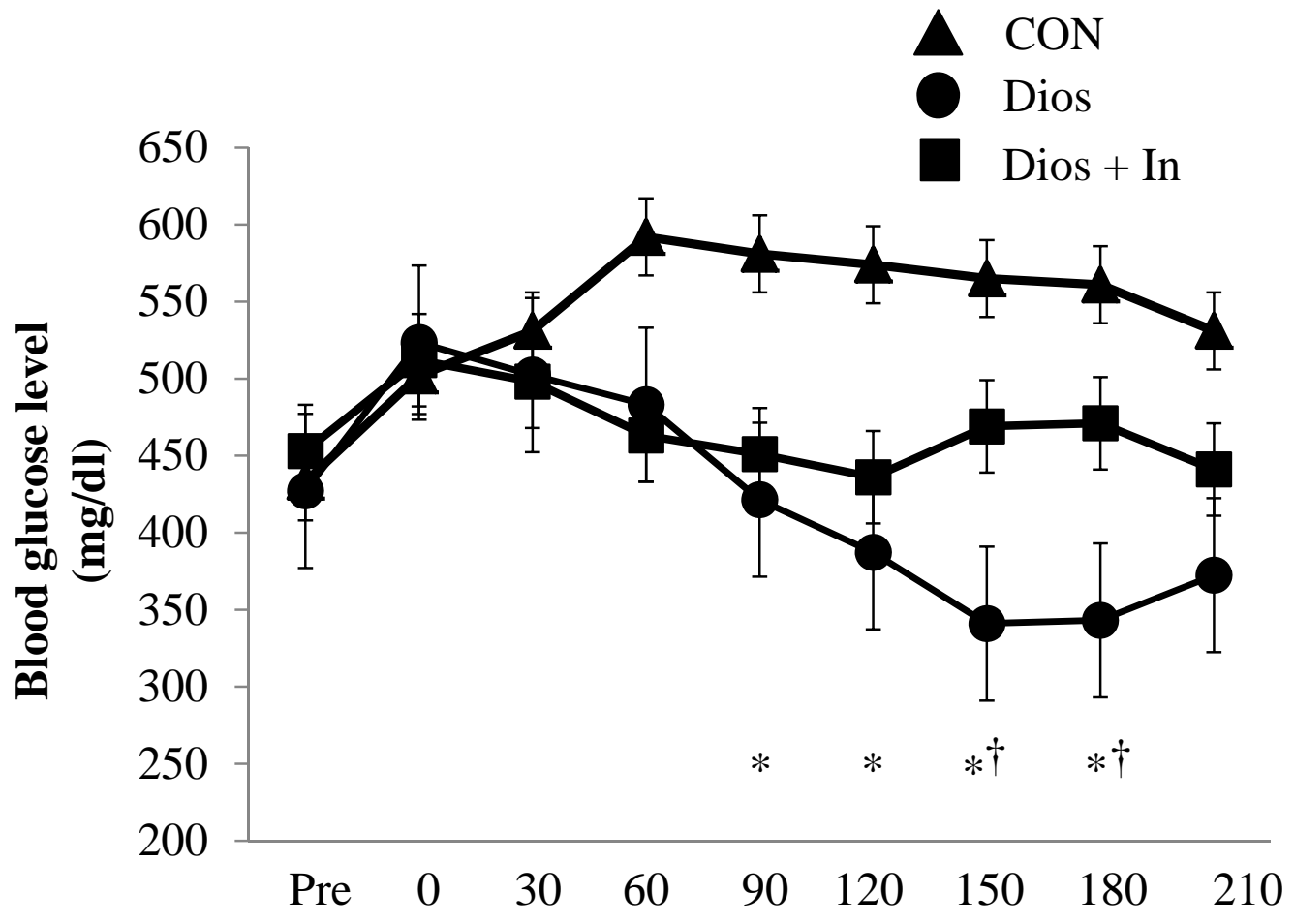


Figure 2

Sato et al.

A

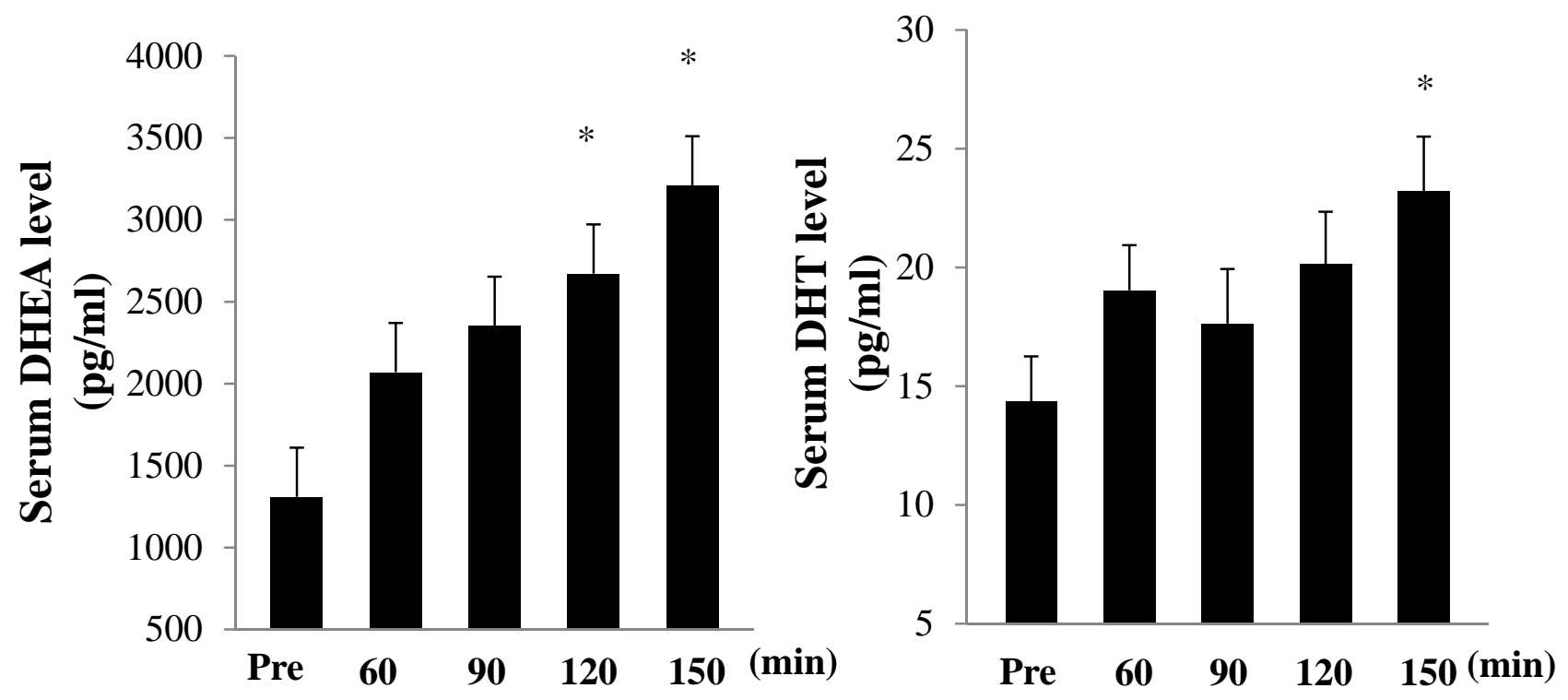

B

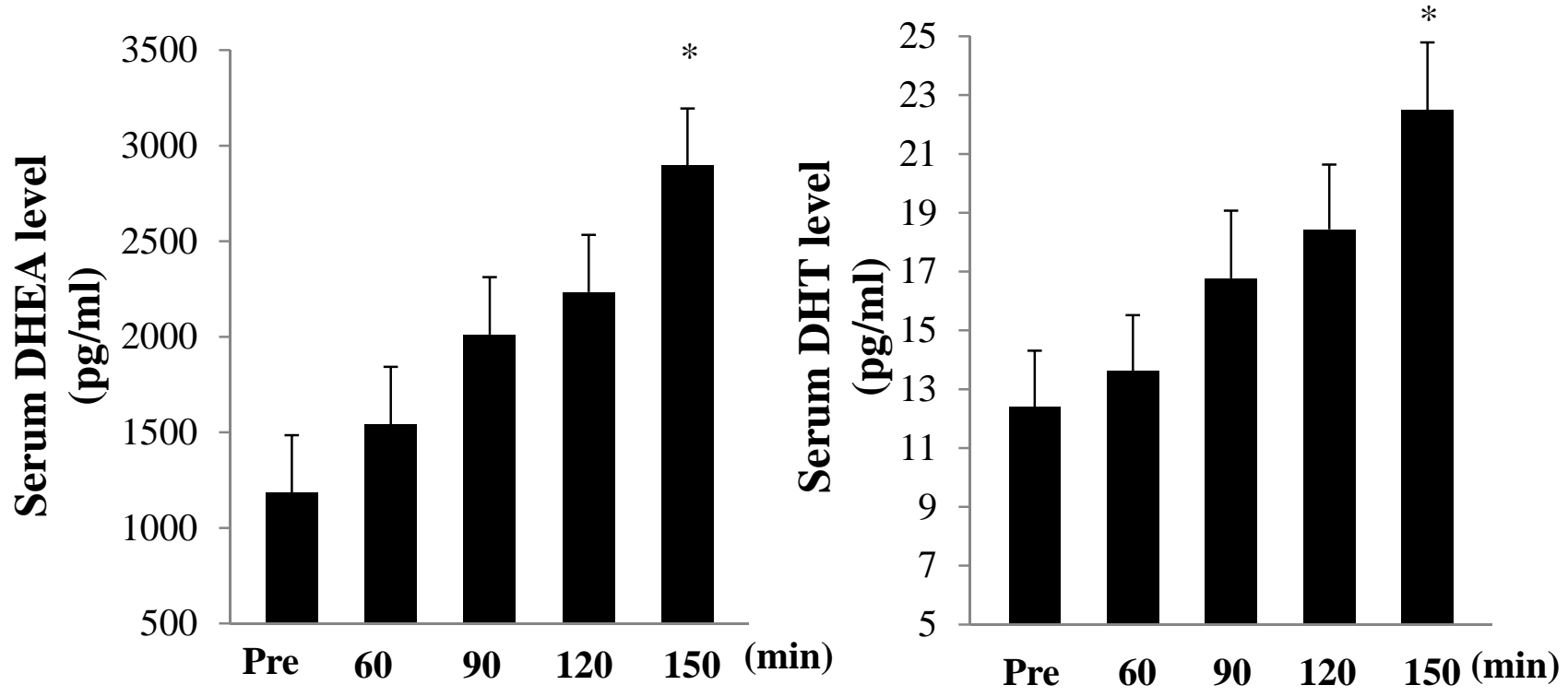


Figure 3

Sato et al.

A
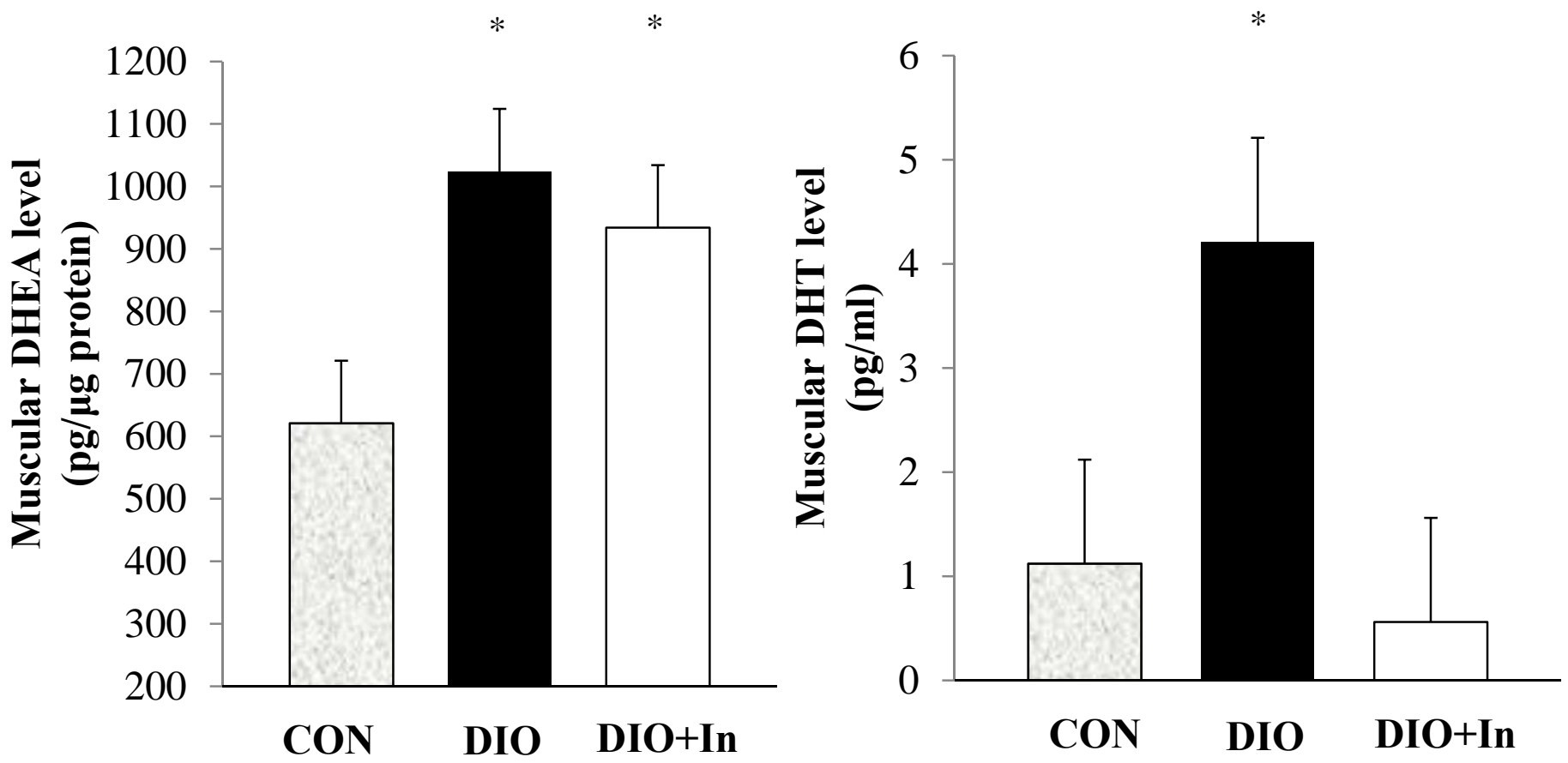

B
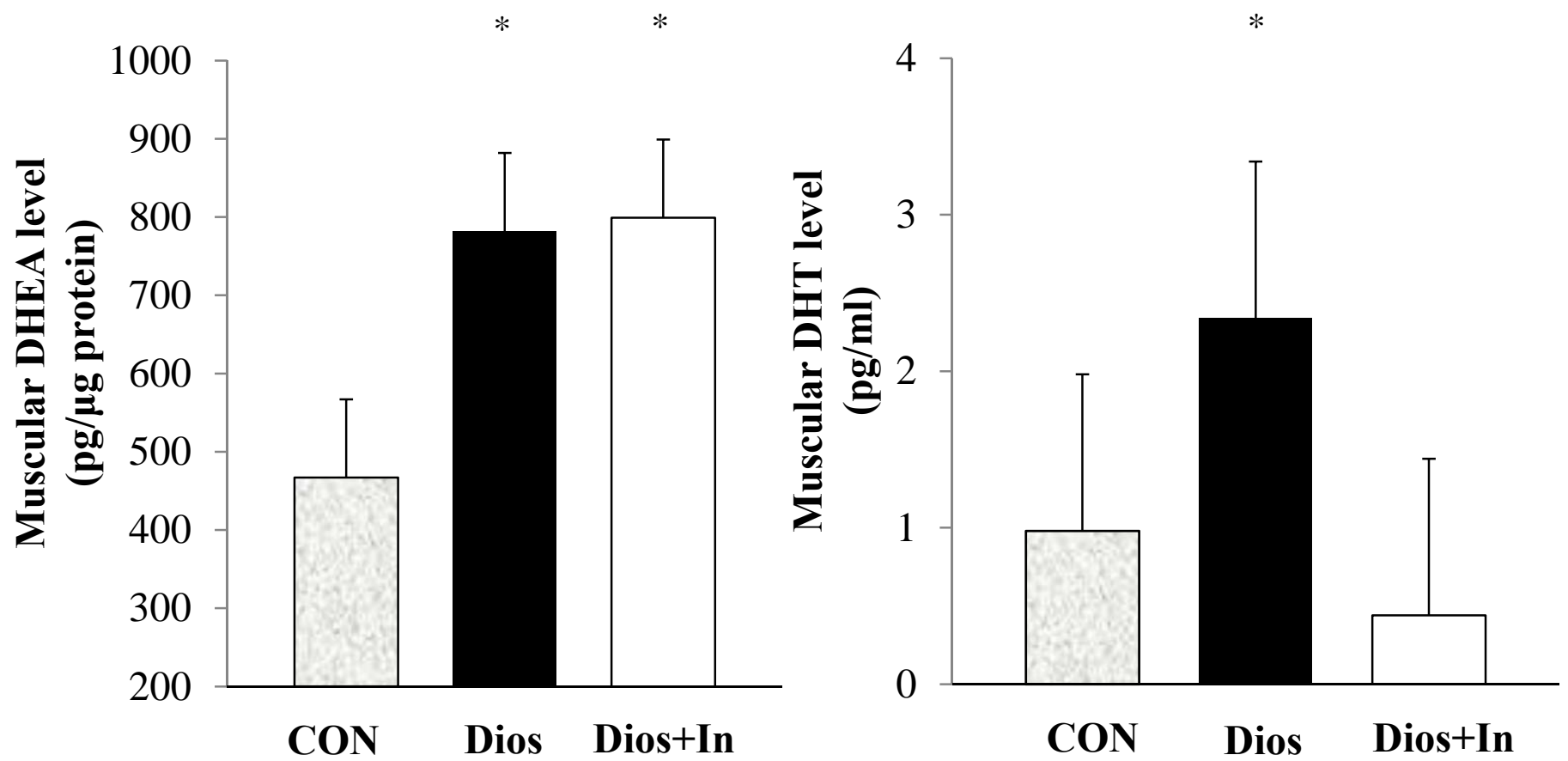
Figure 4

Sato et al.

A

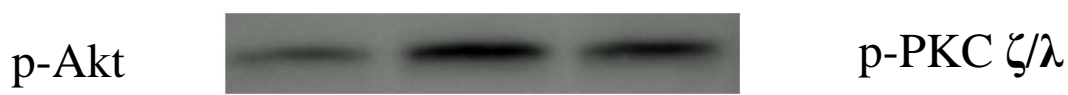

T-Akt
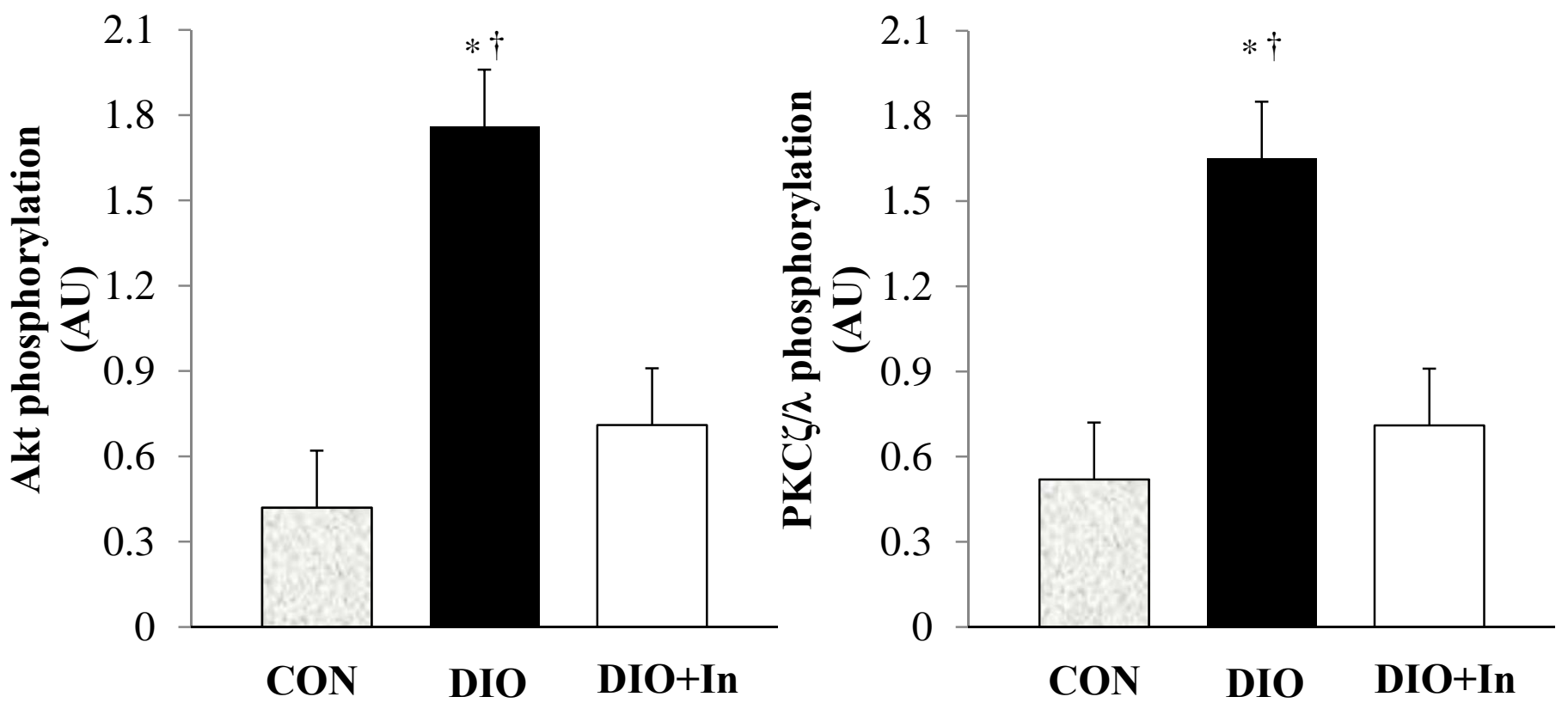

B

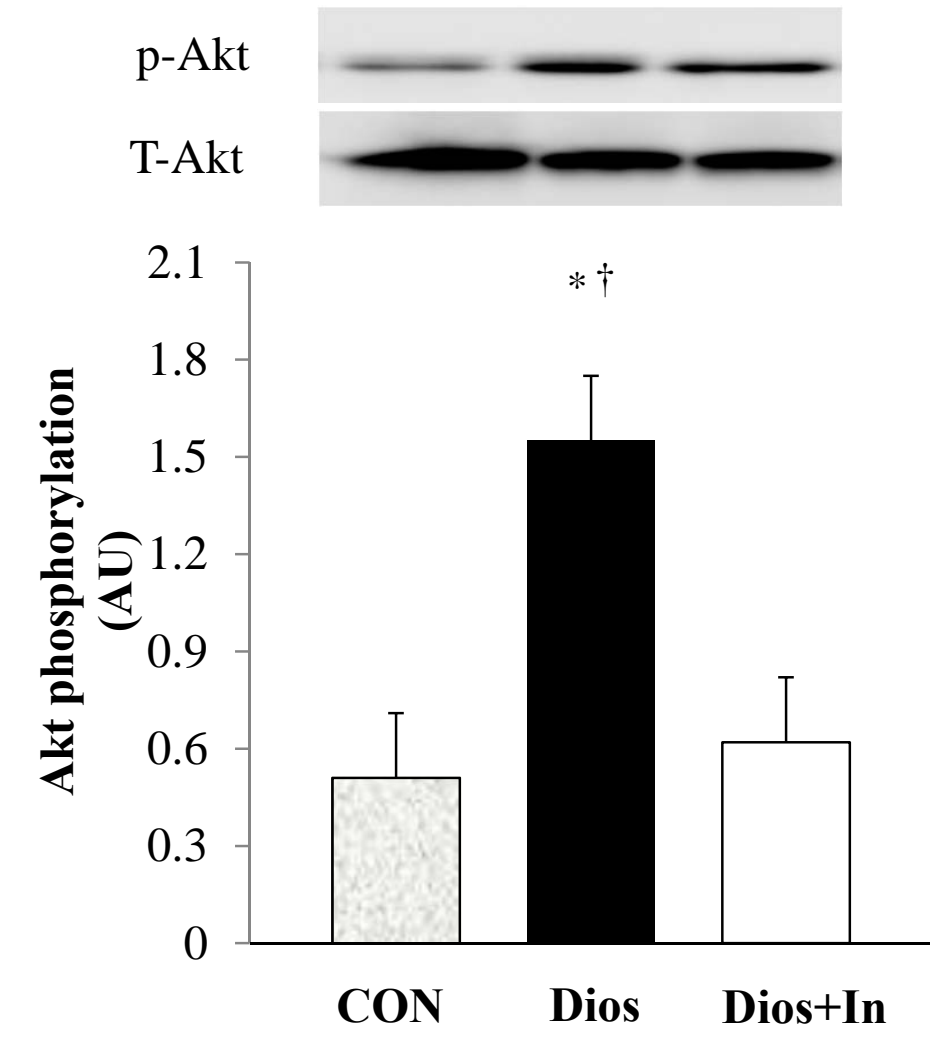

p-PKC $\zeta / \lambda$

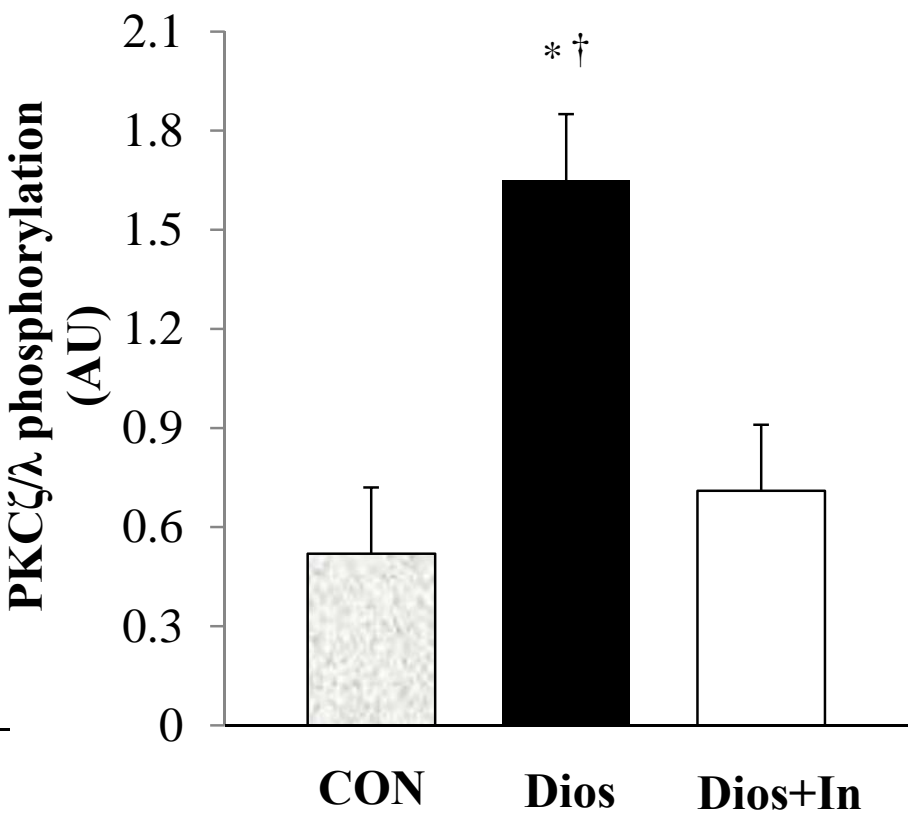


Figure 5

Sato et al.

A

Cytosol

Membrane
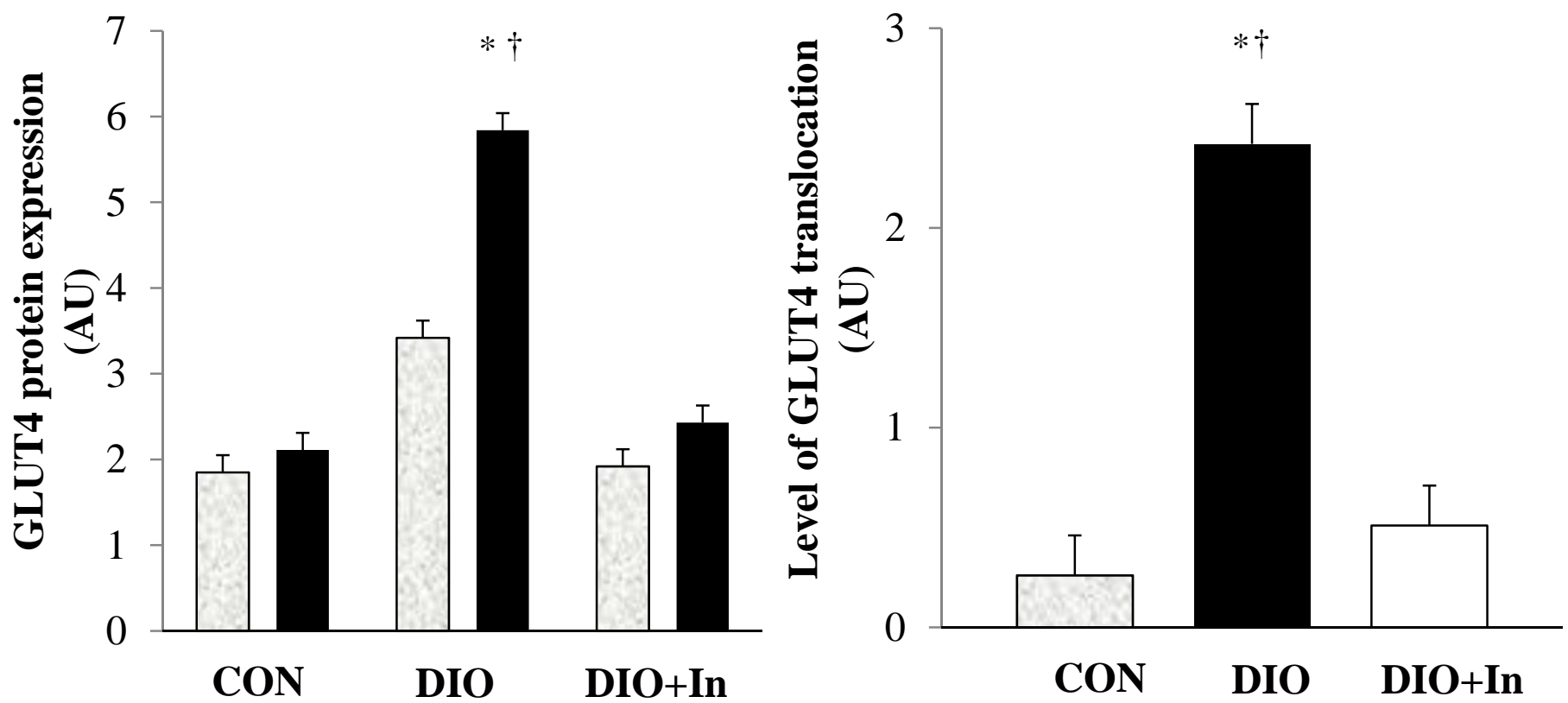

B

Cytosol

Membrane
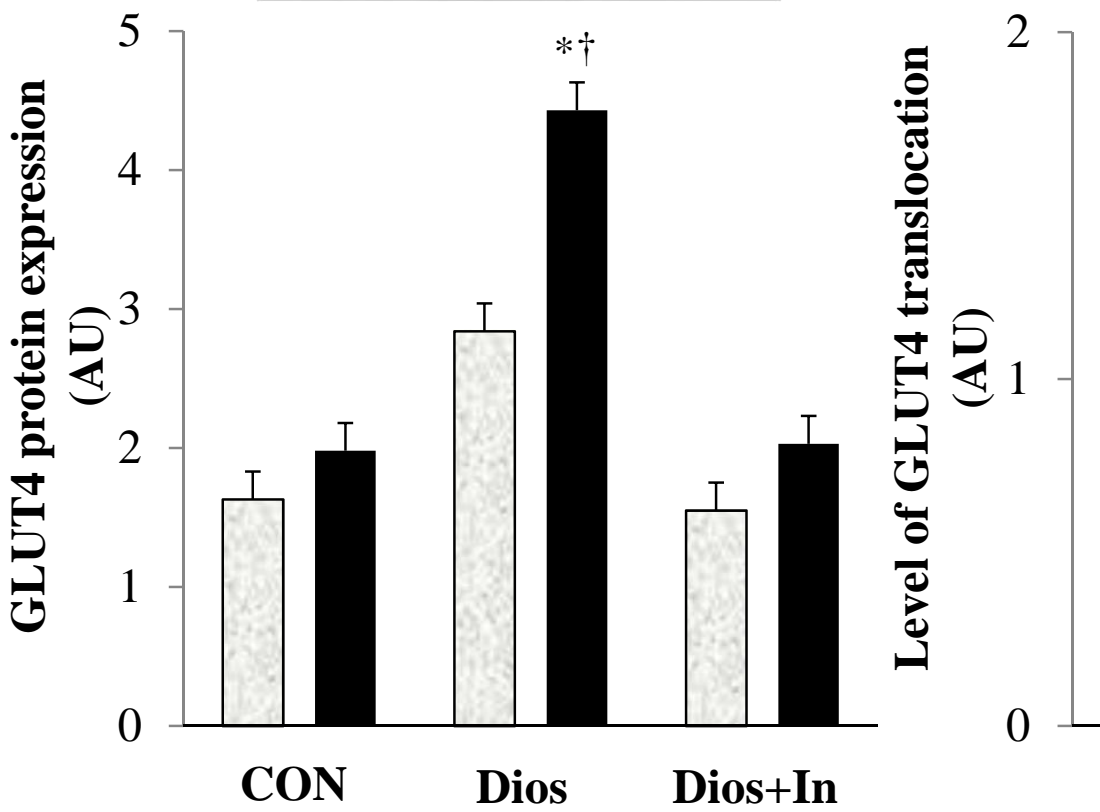

*†

0

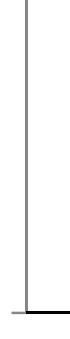

